\title{
WELFARE Y MUTUALISMO EN LA EUROPA CONTEMPORÁNEA: ESTUDIOS SOBRE ESPAÑA E ITALIA
}

\author{
PRESENTATION: \\ WELFARE AND MUTUALISM IN CONTEMPORARY EUROPE: \\ STUDIES ON SPAIN AND ITALY
}

Guillermo Marín*1, Ilaria Pavan ${ }^{2}$

${ }^{1}$ UPV/EHU (España)

${ }^{2}$ Scuola Normale Superiore de Pisa (Italia)

* Correspondencia a: Guillermo Marín. Universidad del País Vasco/Euskal Herriko Unibertsitatea. Facultad de CC. Sociales y de la Comunicación. Departamento de Historia Contemporánea. Barrio Sarriena s/n, Leioa (Bizkaia) 48940 - guillermo. marin@ehu.eus - http://orcid.org/0000-0003-4565-6073

Cómo citar: Marín, Guillermo; Pavan, Ilaria (2019). «Presentación: Welfare y mutualismo en la Europa contemporánea: estudios sobre España e Italia»; Historia Contemporánea, 61, 763-773. (https://doi.org/10.1387/hc.20933).

ISSN 1130-2402 - eISSN 2340-0277 / (C) 2019 UPV/EHU 
Los textos aquí recogidos son resultado del simposio Welfare, previsión social y mundo del trabajo, que tuvo lugar en Vitoria-Gasteiz, en julio de 2017, organizado por el Instituto de Historia Social Valentín de Foronda (Universidad del País Vasco / Euskal Herrriko Unibertsitatea) ${ }^{1}$. El simposio nació de la voluntad de promover un encuentro académico y de solicitar un mayor diálogo entre historiadores españoles e italianos, en lo concerniente a la esfera de la previsión social y las políticas sociales en estos países, buscando dar estímulo a futuros estudios comparados sobre estos objetos de investigación.

En los últimos años han tenido un considerable desarrollo las investigaciones orientadas a analizar elementos comunes de los regímenes franquista y fascista, con una particular atención a los elementos que el primero imitó del segundo y a las afinidades ideológicas entre ambos, o al peso del rol jugado por el catolicismo. Numerosos han sido, asimismo, los estudios que han analizado el papel italiano (tanto fascista como antifascista) en la Guerra Civil española. También la comparación historiográfica relativa al tema de la transición vivida en ambos países - de la dictadura a la democracia-, o el decisivo corte derivado del desenlace de la Segunda Guerra Mundial, han adquirido una nueva centralidad en el diálogo entre historiadores italianos y españoles, así como las investigaciones que han contemplado, en paralelo, a las así llamadas políticas de la memoria elaboradas en ambos paí$\operatorname{ses}^{2}$. Sin embargo, el campo de las variadas fórmulas de previsión social pública/estatal o privada/popular llevadas a cabo antes, durante y después de los años de las dictaduras de Franco y Mussolini, ha permanecido notoriamente ajeno a este reciente desarrollo de las miradas comparadas, suscitando todavía escasa atención entre la comunidad investigadora ${ }^{3}$.

${ }^{1}$ El dossier no incluye todas las aportaciones presentadas y desarrolladas durante el simposio. No están, de hecho, aquí recogidas, las de los trabajos de Margarita Vilar Rodríguez, Rafael Ruzafa Ortega, y Pedro Berriochoa Azcárate.

${ }^{2}$ La producción sobre estos temas es vasta y rica, y no es este el espacio de enumerarla en modo exhaustivo. Señalamos, además, que desde 1992 se publica la revista «Spagna Contemporanea», que ha contribuido intensamente a promover, en la historiografía de España e Italia, los intercambios y las miradas comparadas sobre abundantes aspectos de la historia española en los dos pasados siglos.

${ }_{3}$ Bartolini, Stefania, Gonzalez Canalejo Carmen, «Percorsi di formazione per 1' «infermiera moderna»: Italia e Spagna 1870-1920», en Medicina \& Storia, 11 (2001), 21-22, pp. 329-361; MARÍN, Guillermo, Fernández, Iñaki, «Welfare as totalitarian tool. Fascist Italy and Francoist Spain in the European context», en Ferrán Gallego y Francisco Morente (eds.), The Last Survivor. Cultural and Social Projects Underlying Spanish Fascism, 19311975, Sussex Academic Press, Eastbourne, 2017, pp. 137-155. 
Tanto en España como en Italia no han faltado tanto síntesis como investigaciones específicas sobre el estado social en cada país durante el Ottocento y el Novecento. Ambas historiografías, de hecho, atesoran un largo recorrido en el abordaje de estos temas, habiendo dado lugar a una producción rica y articulada ${ }^{4}$. Pese a la larga trayectoria de ambas historiografías «en solitario», apenas ha habido espacios mutuos de discusión, análisis y comparación, relativos a los acercamientos metodológicos llevados a cabo y a los resultados obtenidos en los estudios de ambos países.

Existen, sin embargo, algunos elementos recurrentes en ambos recorridos nacionales: la tardía industrialización, el peso, en términos económicos y demográficos, de la agricultura, durante parte importante del s. XX, la importancia del rol de la iglesia católica, o la presencia de regímenes dictatoriales prolongados y su confluencia cronológica (decisiva, a nuestro entender, para entender aspectos fundamentales del régimen de Franco hasta el final de la Segunda Guerra Mundial). Estos nexos, aparentemente sustanciales, ponen de relieve el potencial interés que atesora la perspectiva comparada en el estudio de las políticas sociales en España e Italia, en su sustrato cultural e ideológico durante los diferentes momentos políticos de su desarrollo, en sus implicaciones institucionales, en sus resultados concretos (sociales), en su uso propagandístico en clave aglutinadora o nacionalizadora, y en procesos de imitación y transferencia en ambos espacios ${ }^{5}$.

A esta escasa atención dedicada, por la historiografía italiana y española, a los elementos comunes de la previsión social en España e Italia, se ha contrapuesto un decidido interés por parte de los científicos sociales, tradicionalmente más activos en el campo de los estudios sobre el Welfare State. De hecho, a partir de mediados de los años 90 del s. XX se inició un vivo debate en el campo de las ciencias sociales, que ha llevado a teorizar la existencia de aquello que ha sido definido como un «Southern European Welfare State Model», del que formarían parte, junto a Italia y

${ }^{4}$ Remitimos, sobre la abundante bibliografía relativa a la historia del Stato Sociale italiano y sobre el Estado del Bienestar en España, a las referencias bibliográficas detalladas en cada uno de los textos que componen este dossier.

5 Abbott, Andrew, Deviney, Stanley, «The Welfare State as Transnational Event: Evidence from Sequences of Policy Adoption», en Social Science History, 16 (1992), 2, pp. 245-274. 
España, también Portugal y Grecia ${ }^{6}$. Se trata de estudios que se han orientado a poner de relieve los elementos afines en las dinámicas nacionales de estos contextos, tales como: la homogeneidad en la estructura y en el rol de la familia y de la mujer; el desarrollo de iniciativas de protección social estatales muy fragmentadas y sectoriales vinculado al status ocupacional de las diversas categorías de trabajadores (una persistencia, pues, de largo recorrido, influida por la tradición del corporativismo fascista y cuya cimentación provendría de los regímenes de Mussolini, Franco y Salazar); el papel marginal jugado por los entes de asistencia social; la existencia de notables diferencias territoriales en el funcionamiento del sistema de seguridad social, que ha generado, y alimentado, disparidades entre diferentes regiones en cada país; y la existencia de políticas sociales de tipo clientelar, que a menudo han distribuido beneficios a determinados grupos, o categorías de trabajadores, con fines políticos.

En cualquier caso, estos estudios han tendido a analizar, fundamentalmente, las últimas 3 o 4 décadas de la historia de estos espacios nacionales, y en línea con un enfoque muy de ciencias sociales, han propuesto la existencia de un modelo particular de Welfare, o de Welfare regimes, atendiendo relativamente a las peculiaridades históricas de cada país y a las contingencias que han caracterizado cada uno de sus recorridos políticos.

Más allá de las políticas sociales de los Estados (y para el caso de esta publicación, en especial de los Estados español e italiano), pero también en la esfera de la previsión social, este dossier atiende también a aquellas iniciativas asociativas, de matriz privada/popular, destinadas a la protección a los trabajadores ante eventuales infortunios, que anteceden al nacimiento de la preocupación estatal por lo social. Nos referimos a las sociedades de socorros mutuos españolas, y a las società di mutuo soccorso italianas, que dieron sus primeros pasos a caballo entre el primer y el segundo tercio del s. XIX dando lugar a lo que hoy en día se conoce extendidamente como mutualismo. A diferencia de la previsión social patrocinada por lo público, esta constituye una esfera escasamente estudiada, se podría decir que casi desconocida, y que no ha suscitado la atención que este objeto de estudio merece - especialmente entre la historiografía italiana-.

\footnotetext{
${ }^{6}$ Katrougalos, George, Lazaridis, Gabriella (eds.), Southern European Welfare States, Palgrave Macmillan, London, 2003; Moreno, Luis, «The model of social protection in Southern europe. Enduring Characteristics?, en Revue française des affaires sociales , 2006, 5 , pp. 73-95; Gal, John, «Is there an extended family of Mediterranean welfare states?», en Journal of European Social Policy, 2010, 4, pp. 283-300.
} 
Para el caso de España, y en contraste con los abundantes estudios sobre mutualismo decimonónico ${ }^{7}$, durante el $\mathrm{s}$. XX $-\mathrm{y}$ en especial durante la dictadura franquista - el mapa del asociacionismo mutualista se torna difuso, complejo de rastrear ante la enmarañada interacción que caracterizó a la esfera de lo dictatorial respecto a la esfera de lo voluntario ${ }^{8}$. Si miramos hacia Italia, los estudios que se han acercado al campo del asociacionismo durante el fascismo han insistido en los estrechos márgenes de desenvolvimiento autónomo de la sociabilidad al margen de la batuta política/sindical, si bien en algunas esferas el aparataje político fascista habría tenido que lidiar con la Iglesia, y con el papel de estructuras vinculadas a ella como Azione Cattolica, fuertemente arraigadas en espacios como el educativo9. Aspectos relativos a la estructuración de lo administrativo en general, y de la vocación absorbente del fascismo en todo aquello que irradiara asociacionismo en particular, como la pretendida nacionalización del tiempo libre a través de la «Opera Nazionale del Dopolavoro», han sido abundantemente estudiados ${ }^{10}$. Los paralelismos con la Obra Sindical Educación y Descanso, que de hecho será una imitación del modelo italiano anteriormente mencionado, resultan reveladores, siendo también similar la batería de actividades que se coordinaron para «entretener encuadrando» ${ }^{11}$.

Las reflexiones de la historiografía italiana sobre el mutualismo durante el fascismo han tendido a moverse en los planteamientos de una absorción del tejido mutualista italiano por el entramado sindical del Partito Unico Fascista que habría «borrado de un plumazo» toda la herencia mutualista anterior ${ }^{12}$. Surge, en cualquier caso, la duda, de si el asociacio-

7 Remitimos al capítulo de Montserrat Duch Plana que forma parte de este dossier.

8 Maza Zorrilla, Elena, «Panem et circenses». Cultura asociativa durante el franquismo», en Alcores, 6, 2008, pp. 83-112.

9 Ridolfi, Maurizio, «Asociacionismo, mundo del trabajo y culturas populares en la Italia fascista», Alcores, 6, 2008, pp. 21-44.

10 De Grazia, Victoria, Consenso e cultura di massa nell'Italia fascista. L'organizzazione del dopolavoro, Laterza, Bari-Roma 1981; Inaudi, Silvia, «Le politiche assistenziali nel regime fascista», en Paolo Mattera (ed.), Momenti di Welfare in Italia. Storiografia e percorsi di ricerca, Viella, Roma 2012.

11 López Gallegos, Silvia, «El control del ocio en Italia y España. De la Opera Nazionale Dopolavoro a la Obra Sindical de educación y Descanso, Investigaciones históricas: Época moderna y contemporánea, n. ${ }^{\circ} 24,2004$, pp. 215-236.

${ }^{12}$ Fabio Bertini, Il fascismo dalle assicurazioni per i lavoratori allo Stato sociale, en Marco Palla (ed.), Lo Stato fascista, Milano. La Nuova Italia, 2001, pp. 177-313; Silei, Gianni (ed.), Volontariato e mutua solidarietà. 150 anni di previdenzain Italia, Lacaita, Roma-Bari-Manduria, 2011. 
nismo mutualista de matriz popular, aquel que hundía sus raíces en un contexto anterior al de la acción social estatal, hubiera podido seguir funcionando en la Italia fascista, lejos del escrutinio del aparataje políticoadministrativo del régimen, y lejos también, por lo tanto, de quedar reflejado en fuentes oficiales, mediante iniciativas mutualistas poco visibles y quizá escasas, urbanas o agrarias. Como, de hecho, ha podido constatarse para el caso de la España franquista en algún estudio local ${ }^{13}$. En esta línea, algunos estudios locales en clave italiana han puesto en duda la capacidad del fascismo para afirmar un nuevo modelo de agregación social, sustitutivo de la tradicional solidaridad popular y de las prácticas de grupo que asomaban no sólo en los lugares de trabajo, sino sobre todo en la cotidianidad de la vida familiar y comunitaria más inaccesibles a la capacidad controladora y aglutinante del fascismo ${ }^{14}$.

Como podrá observarse, en las miradas comparadas hacia el mutualismo español e italiano se parte de una plataforma en la que la realización de ejercicios comparativos es muy compleja en tanto que son muy escasos los estudios sobre la materia. Pero no por ello deja de ser un campo de estudio de gran interés. Los paralelismos entre el franquismo y el fascismo asoman en la aparente evidencia de que ambos supusieron, a su manera, un certero golpe que hizo languidecer el modelo de sociedades de socorros mutuos que venía del s. XIX. El fascismo se propuso acabar con él, como lo hizo con cualquier otra iniciativa asociativa que no estuviera directamente controlada por el Estado. Mientras, y para el caso del franquismo, el mutualismo de matriz popular siguió vivo durante la dictadura, aunque hubo de transformarse a intensidad variable, para poder quedar enmarcado en un espacio legal reconocido, el Reglamento de Montepíos y Mutualidades de 1943. Este cuerpo legal se puso en marcha para organizar la esfera de lo mutualista, controlándola y limitándola. Este control se plasmó en una reglamentación pretendidamente férrea en aspectos no solo políticos, sino también, económicos, técnicos y actuariales, que en la práctica hizo desaparecer a muchas de las sociedades de socorros mutuos de más viejo cuño. Otras, sin embargo, fueron capaces de adaptarse, reciclándose, y dando lugar al surgimiento de lo que podría denominarse un

13 Marín, Guillermo, Mutualismos. La previsión social en Álava durante el franquismo, UPV/EHU, Bilbao, 2017

${ }^{14}$ Grana, Daniele, «La prevenzione del disenso: la política assistenziale del fascismo», en Bertucelli y Margagnoli (eds.), Regime fascista e società modenense, Mucchi, Modena, 1995, pp. 121-140. 
nuevo tipo de mutualismo, respecto al anterior a la reglamentación franquista.

El peaje a satisfacer para este «nuevo mutualismo» en España emparentaba con una visión que, como para el caso del fascismo en Italia, quiso destruir toda opción política que no comulgara (o que fuera sospechosa de no comulgar) con los postulados del nuevo Estado. En el campo del mutualismo, esta vocación se materializó en un férreo control político-social (a través de un constante control de los antecedentes políticos de los miembros de las juntas directivas de las entidades mutualistas), de depuraciones, y de la inevitable desaparición, desnaturalización, o control especialmente intenso, de aquellas entidades de previsión que tuvieran, o hubieran tenido, algún tipo de matiz político.

Puede decirse, por lo tanto, que queda mucho terreno por explorar en el vasto campo de la previsión social en España e Italia, en clave comparada, tanto en su vertiente público/ estatal como en aquella privada/popular. Donde comparar, haciendo nuestras las enseñanzas, antiguas pero vigentes, de March Bloch, significa sobre todo establecer diferencias entre fenómenos similares que hagan asomar, fundamentalmente, la originalidad y las peculiaridades de las respectivas experiencias.

En este sentido, los capítulos de Ilaria Pavan y de Andrea Rapini ofrecen un primer punto de reflexión. Ambos evidencian, de hecho, como en el caso italiano el contexto de la Primera Guerra Mundial supuso un momento decisivo - una suerte de «cantera», podría decirse - para la promoción y la puesta en marcha, a efectos prácticos, de un sistema moderno de políticas sociales, en línea con los de otros países europeos. Las nuevas exigencias derivadas del conflicto (en al ámbito sanitario, asistencial, previsor, y para esferas como la orfandad o el ámbito de los veteranos y mutilados de guerra) llevaron a los gobiernos italianos a un inédito dinamismo, no exento de limitaciones y contradicciones, y con un claro trasfondo orientado a reforzar el control social. Nuevas lecturas sobre el Welfare State en clave internacional, de origen politológico, pero que recientemente han suscitado atención en el campo de la historiografía, vienen resaltando que las guerras totales del s. Xxrepresentan una página decisiva, y a menudo un punto de inflexión, en la promoción de políticas sociales en muchos contextos nacionales, tanto europeos como extraeuropeos. Durante largo tiempo, la guerra no ha constituido, de hecho, un campo de investigación importante en el estudio del camino evolutivo del Welfare State, que a menudo ha tendido a vincularse más con el surgimiento y desarrollo de reivindicaciones sociales y sindicales, o a plan- 
tearse como un aspecto paralelo al de procesos de modernización en clave democrática. Sin embargo, un número creciente de investigaciones se centra en el papel desempeñado por los dos grandes conflictos mundiales del pasado siglo XX en la historia del Welfare, en relación con las profundas transformaciones en la estructura y en el papel de los Estados que, a menudo, han implicado las guerras en el marco europeo (aunque no sólo) ${ }^{15}$.

En este sentido, la lejanía tanto de España como de Portugal respecto a esta variable, y la evolución de la política social en ambos países en el marco de este contexto, puede constituir un elemento de particular interés que parece no ajustarse dentro del paradigma explicativo del Welfare-Warfare propuesto recientemente para encuadrar históricamente el desarrollo de diversos sistemas de seguros sociales ${ }^{16}$. Del mismo modo, podría ser útil profundizar en el análisis del papel que jugó la guerra civil española en el desarrollo de las políticas sociales en España, tomando como referencia algunos de los notables acercamientos que, de forma más o menos directa, aportan claves reseñables sobre este aspecto ${ }^{17}$.

También los capítulos de Chiara Giorgi y de Guillermo Marín, dedicados al fascismo y al franquismo, y a un análisis comparado del rol institucional y propagandístico de los dos mayores entes gestores de las políticas sociales en España e Italia - el Instituto Nacional de Previsión (INP) y el Istituto Nazionale Fascista della Previdenza Sociale (INFPS)-, hacen asomar tanto notorias similitudes como importantes diferencias. Si las políticas sociales fueron, para ambos regímenes, vehículos de primer orden mediante los cuales intentar construir y consolidar consensos mediante mecanismos tanto instrumentales como identitarios (la construcción de la imagen de una colectividad nacional sana, «remodelada» por el régimen y acorde con sus valores), el INFPS fue usado también como recurso mediante el cual financiar otras políticas, como aquellas destinadas a la colonización de Libia, en línea con la idea fascista del impero in primis. La utilización, para este tipo de fines, de los fondos gestionados por los entes de previsión creados por el

15 Obinger, Herbert, Petersen, Klaus, Starke, Peter, Warfare and Welfare. Military Conflict and Welfare State Development in Western Countries, Oxford Up, Oxford, 2018.

16 Véanse los capítulos de Ilaria Pavan y Andrea Rapini, en este dossier.

${ }_{17}$ Molinero, Carmen, La captación de las masas. Política social y propaganda en el régimen franquista, Cátedra, Madrid, 2005; Cenarro, Ángela, A Social Work in the Spanish Civil War, en Matthews, James (ed.), Spain at war. Society, culture and mobilization 19361944, London, Bloomsbury, 2019, pp. 141-158. 
fascismo (el INFPS, fundamentalmente), es pues una característica distintiva entre ambos regímenes, derivada en parte de inquietudes de proyección exterior no homologables. Al mismo tiempo, si la narrativa propagandística usada por ambos regímenes tuvo evidentes vasos comunicantes (construcción, mediante ideología pero también mediante leyes, de un modelo de familia determinado basado en el ideal del hombre asalariado y de la mujer ama de casa, políticas marcadamente pronatalistas), el caso italiano se caracteriza por una torsión final, a finales de la década de los 30, abiertamente racista y antisemita, en línea con la adopción, a partir de 1937-38, de políticas discriminatorias respecto a hebreos y súbditos coloniales.

En lo que respecta al capítulo de Montserrat Duch, su texto se centra en aquella otra variante de la previsión social: la popular, aquella mutualista basada en las sociedades de socorros mutuos y en la reciprocidad, y en un espacio, Cataluña, que la historiografía española describe como el vergel del mutualismo español por la cantidad, variedad y dinamismo de iniciativas mutualistas que allí surgieron y se desarrollaron. La cronología del estudio de Duch llega hasta 1936, año de corte tras el cual nuestro conocimiento sobre el mutualismo catalán se torna algo más opaco, abriendo una rica vía de investigación en absoluto exenta de hilos de los que sería interesante tirar, y de fuentes sobre las que sería interesante profundizar.

En este texto se aprecian interesantes reflexiones que los estudios venideros sobre mutualismo, tanto aquellos en clave comparada España/Italia, como aquellos que no aspiren necesariamente a ser comparativos, habrían de tener en cuenta. La primera tiene que ver con la situación de la mujer y su posición tanto en los mercados laborales como en el ámbito de marcos asociativos como el mutualismo. Durante la segunda mitad del s. XIX y el primer tercio del s. Xx, su escasa (o prácticamente nula) visibilidad legal contrastaba con su sólida y muy abundante presencia numérica dentro de muchos espacios laborales en los que, también, lo mutualista se hallaba presente. Puede decirse, que tras 1936 la posición de la mujer no sólo no cambiará, si no que empeorará, al menos si atendemos al entramado legal laboral construido por el franquismo, en el que se trató, a veces con más éxito, otras con menos, de sacar a las mujeres de talleres y fábricas, y situarlas en un plano de dependencia económica y de sometimiento tutelar, así como en un plano muy secundario de su sistema de seguros sociales.

Otro de los elementos imprescindibles de su texto, y necesarios para una comprensión lo más amplia posible del fenómeno mutualista, es el campo de los salarios, y su relación con la mayor o menor capacidad de asumir los «costes básicos de vida» entre grupos laborales de diferente 
tipo. La reflexión nos lleva a entender el estudio del mutualismo, y el grado de adhesión popular que tuvo, no sólo como una cuestión de querer, o no, formar parte de algo: si no, también, como una cuestión de capacidad de ser previsor. De poder, o de no poder, pagar una pequeña cuota periódica para la cobertura de contingencias como la muerte o la enfermedad, que podían ser dramáticas para el núcleo familiar, cuando quizá, en algunos casos, la economía familiar a duras penas llegaba a cubrir las necesidades cotidianas más básicas. En este sentido, las aportaciones de Margarita Vilar, desde la Historia Económica, sobre salarios y costes de vida durante el primer franquismo, resultan imprescindibles para dotar de plataformas de estudio al análisis de esta dimensión ${ }^{18}$.

No puede dejar de ser mencionado, además, el tremendo potencial para la investigación que se adivina en la Base de dades de l'Associacionisme català contemporani manejada por Montserrat Duch. El estudio realizado por Duch, y que llega hasta los años 30 del siglo pasado, podría y debería tener una continuación cronológica que nos ayude a comprender el fenómeno del mutualismo catalán durante el franquismo, y así confirmar (o si procediera, matizar), este aparente languidecimiento del mutualismo durante el franquismo, más allá de los años 40 del s. XX.

Con un conjunto de fuentes sólido, el texto de Duch dibuja un mutualismo catalán vivo, ágil, y dinámico, en el que también encontramos un número importante de Sociedades de Socorros Mutuos de estructura técnicas muy básicas y por lo tanto, más vulnerables frente a contingencias como el envejecimiento de sus asociados. El corte decisivo, en el análisis del número de sociedades de socorros mutuos en Cataluña entre 1890 y 1940, donde parece comenzar a precipitarse el mundo del mutualismo, se produce, muy evidentemente, entre 1931 y 1940 . En este periodo, y a modo ilustrativo, en Barcelona solo se crearon 62 nuevas entidades (frente a las 438 creadas en el periodo 1911-30), o en Girona solo 5, frente a las 50 del periodo anterior. Sin duda este gran descenso debió tener lugar debido múltiples factores. Entre ellos, se estima oportuno mencionar de nuevo la gestión de lo mutualista llevada a cabo por el franquismo, que a buen seguro supuso al punto y final de muchas entidades arcaicas, poco tecnificadas, de viejo cuño, o políticamente sospechosas.

18 Vilar Rodríguez, Margarita, «Estrategias de supervivencia de las familias trabajadoras en el marco laboral hostil de la posguerra civil española (1939-1958)», en Sociología del trabajo, n. ${ }^{\circ}$ 56, 2006, pp. 119-154. 
Queda, en cualquier caso, la cuestión (y es una cuestión que nosotros, la comunidad historiográfica, habremos de escrutar) de qué pasó con el mutualismo catalán a partir de los años 40 del s. XX, para poder complementar este conocimiento con el que nos proporciona Duch, y con el que nos han dado, en el ámbito catalán, los abundantes y ricos estudios sobre espacios y ámbitos laborales concretos de la Cataluña del s. XIX ${ }^{19}$. Ello nos llevaría a saber si la inflexión del mutualismo catalán fue concluyente, o si sobre la plataforma legal mutualista construida a principios de los años 40 , surgió un nuevo mutualismo, distinto del anterior y adaptado a las exigencias controladoras y coercitivas del franquismo... pero que, a nuestro entender, no dejaba de ser mutualismo, aunque lo fuera de otro modo.

Además de su riqueza de contenidos, el texto de Montserrat Duch abre un fértil terreno, en lo metodológico, para estudios sobre mutualismo en otras regiones de España o en otros países como Italia, y no sólo en su cronología de trabajo en este texto, sino también en posteriores a los años 40 , constituyendo una hoja de ruta muy a tener en cuenta en futuras investigaciones.

\section{Financiación}

Este trabajo forma parte de las investigaciones desarrolladas en el marco del proyecto «Nacionalización, Estado y violencias políticas (siglos XIX-XXI)», apoyado por el Ministerio de Economía y Competitividad (HAR2017-83955-P), por el Gobierno Vasco (IT 1227-19) y por la Universidad del País Vasco (UPV-EHU) (GIU 18/107)

19 Remitimos al capítulo de Montserrat Duch, que forma parte de este dossier. 